Asian Journal of Information Technology 10 (3): 119-121, 2011

ISSN: $1682-3915$

(C) Medwell Journals, 2011

\title{
Additive White Gaussian Noise Removal Using Viterbi Algorithm
}

\author{
M. Sreedevi and P. Jenoaul \\ Anna University, Tamil Nadu, India
}

\begin{abstract}
Noise removal in images is one of the most basic tasks of image processing. Algorithms for removing noises in image sequences aim to remove the additive noise while utilizing both the spatial and the temporal domains. Such an approach is expected to lead to a gain both in the denoising performance and the computational load when compared to applying a single image denoising algorithm to each image separately. We propose a new algorithm viterbi by considering 3-D (spatio-temporal) patches, a propagation of the +dictionary over time and averaging that is done on neighboring patches both in space and time. As the dictionary of adjacent frames is expected to be nearly identical, the number of required iterations per frame can be significantly reduced.
\end{abstract}

Key words: Denoising, viterbi, sparse representation, video, filters, India

\section{INTRODUCTION}

Denoising of images is also been termed as noise removal which aim to recover a high quality signal from a degraded version of it. Denoising image sequences extends the above task to handle the temporal dimension as well (De Haan, 1999). Such sequences can be TV broadcast, camcorder files and more. In many cases, one can assume the noise to be an additive zero-mean white Gaussian noise as common also in the still image denoising literature. These desired and expected gains emerge from the high temporal redundancy in image sequences. Indeed, in many cases image sequences are noisier than single images due to the high capture rate making the use of the temporal dimension that much more important. Many problems in image processing and computer vision are in a dire need for prior models of the images they handle. Spatial redundancy is correlation between neighboring pixel values. Temporal redundancy is correlation between adjacent frames in a sequence of images (in video applications). The problems related to pixel signal decomposition and reconstruction is main hindrances for effective denoising (Elad and Aharon, 2006).

In many cases, image sequences are noisier than single images due to the high capture rate. Furthermore, denoising process is time consuming for signal decomposition and reconstruction using selected method of thresholding to suppress undesirable signal components in various biomedical signal and image processing Applications. Lesser Peak Signal to Noise Ratio (PSNR) which is the measure of quality of the denoising result versus the original signal is also a challenge in number of applications.
This study can be dividing into four modules. The first module deals updating sparcity and redundancy representation. The second concerns implementing viterbi algorithm (Kuo and Lin, 1996). The third deals with parameter selection and performance evaluation.

\section{REVIEW OF BASIC SYSTEM}

A lot of existing methods are available for denoising an image signal. The sparse representation algorithm denoises the image while simultaneously train a dictionary on its (corrupted) content using the Viterbi algorithm. The dictionary training algorithm is limited in handling small image patches (Siu and Chan, 2006). In Gaussian scale mixtures each neighborhoods of coefficients at adjacent positions and scales are modeled as the product of two independent random variables: a Gaussian vector and a hidden positive scalar multiplier by Aharon et al. (2006).

Spatiotemporal kalman filter: It needs 3D-AR model for the image sequence and the dimensionality of the state vector at each pixel is high. So it increases the amount of computation as well as storage required for processing each frame.

Temporal motion compensated adaptive linear MMSE filter (Boyce, 1992): Two pixels which is in past and future along motion trajectory of this pixel are used to compute local station (Zlokolica et al., 2004).

Spatial adaptive linear MMSE filter: Algorithm has high computation cost and four motion vectors (Jansen, 2001) need to be stored for each pixel.

Corresponding Author: M. Sreedevi, Anna University, Tamil Nadu, India 
Adaptive weight averaging filter: In adaptive weight averaging filter, pixel in spatio temporal is neighborhood (Elad and Protter, 2009). If the pixels have same intensity is kept aside.

Wavelet domain video denoising: Recursive temporal filtering and spatial filtering in Brailean et al. (1995), Rajpoot et al. (2004) and Pizurica et al. (2003) is used. Temporal filter and then spatial filtering is done. Without introducing spatial filter, reducing spatial blur in denoised image sequence because if reliable motion vector is not found, they reduce the amount of temporal recursive filtering. So error will not occur in iteration.

Image denoising using sparsity and redundancy: Denoising of image sequences that are corrupted by zero-mean additive white Gaussian noise. Relative to single image denoising techniques, denoising of sequences aims to also utilize the temporal dimension. This assists in getting both faster algorithms and better output quality. It focuses on utilizing sparse and redundant representations for image sequence denoising. In the single image setting, the viterbi algorithm is used to train a sparsifying dictionary for the corrupted image.

Some modifications lead to substantial benefits in complexity and denoising performance, compared to (Aharon et al., 2006) simply running the single image algorithm sequentially. They are the atoms used are 3-D; the dictionary is propagated from one frame to the next, reducing the number of required iterations and averaging is done on patches in both spatial and temporal neighboring locations.

\section{PROPOSED METHODOLOGY}

Viterbi algorithm may reduce the noise in the trellis search. It is used to eliminate the sequences as new data received. Since the signal memory is one bit which we denote $\mathrm{L}=1$, the trellis reaches its regular form after two transitions. At $\mathrm{t}=2 \mathrm{~T}$, there are two signal paths entering each of the nodes and two signal paths leaving each node. The two paths entering the node. So at $t=2 \mathrm{~T}$ corresponds to the information bits $(0,0)$ and $(1,1)$. For two paths entering node so the two Euclidian distance:

$$
\begin{aligned}
& D_{0}(0,0)=\left(r_{1}+\sqrt{E_{b}}\right)^{2}+\left(r_{2}+\sqrt{E_{b}}\right)^{2} \\
& D_{0}(1,1)=\left(r_{1}-\sqrt{E_{b}}\right)^{2}+\left(r_{2}+\sqrt{E_{b}}\right)^{2}
\end{aligned}
$$

By using the outputs $r_{1}, r_{2}$. The viterbi algorithm compares these two metrics and discards the path with the lower metric is saved and it is called as the survivor at $\mathrm{t}=2 \mathrm{~T}$. The elimination of one of the two paths may be done without compromising the optimality of the trellis search because any extension of the path with the larger distance beyond $\mathrm{t}=2 \mathrm{~T}$ will always have a larger metric than the survivor that is extended along the same path $\mathrm{t}=2 \mathrm{~T}$. The two metrics are compared and the signal path with the larger metric is eliminated. The signal paths at nodes $\mathrm{S}_{0}, \mathrm{~S}_{1}$ are the extended along the two survivor paths:

$$
\begin{aligned}
& D_{1}(0,1)=\left(r_{1}+\sqrt{E_{b}}\right)^{2}+\left(r_{2}-\sqrt{E_{b}}\right)^{2} \\
& D_{1}(1,0)=\left(r_{1}-\sqrt{E_{b}}\right)^{2}+\left(r_{2}-\sqrt{E_{b}}\right)^{2}
\end{aligned}
$$

The two metrics are compared and the path with the larger metric is eliminated. Thus the viterbi algorithm computes two metrics for the two signal paths entering a node at each stage. Therefore, the number of paths searched is reduced. Thus a variable delay in bit or symbol detection is avoided and the noise in the performance is reduced. Denoised Image using Viterbi and the adaptive dictionary Image are shown in Fig. 1 and 2.

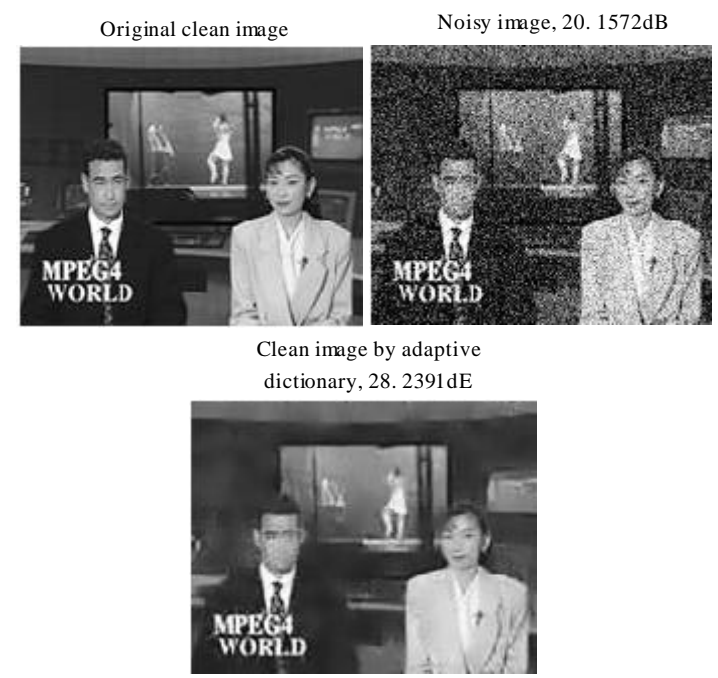

Fig. 1: Denoised image by using viterbi

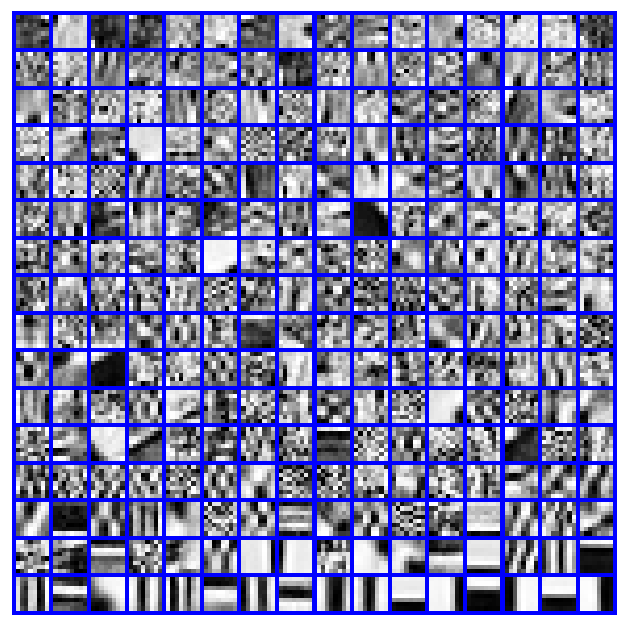

Fig. 2: Adaptive dictionary 
Table 1: Results of the proposed algorithm compared to DCT and Global trained dictionary

\begin{tabular}{lccccc}
\hline $\begin{array}{l}\text { Test } \\
\text { sequence }\end{array}$ & $\begin{array}{c}\text { Input } \\
\text { noise }\end{array}$ & $\begin{array}{c}\text { Frame } \\
\text { level }\end{array}$ & $\begin{array}{c}\text { Results from } \\
\text { index }\end{array}$ & $\begin{array}{c}\text { Results from } \\
\text { global trained } \\
\text { in dB }\end{array}$ & $\begin{array}{c}\text { Results from } \\
\text { Viterbi in dB }\end{array}$ \\
\hline News & 25 & 3 & 27.6918 & 27.8931 & 28.2391 \\
Tennis & 25 & 3 & 26.1324 & 26.8756 & 28.7645 \\
Garden & 25 & 3 & 25.7843 & 27.1201 & 30.2312 \\
\hline
\end{tabular}

The peak signal to noise ratio which is noise power and chosen sequences for denoising obtained in image sequence denoising using Viterbi algorithm is shown in Table 1.

Performance metrics: Two of the error metrics used to compare the various image denoising techniques is the Mean Square Error (MSE) and the Peak Signal to Noise Ratio (PSNR). Another metrics has to be mentioned, they are temporal dimension and size of the video.

\section{CONCLUSION}

The extension of this basic algorithm to handle image sequences is discussed both on the mathematical level and in practical terms. Three extensions are proposed. They are the use of spatio-temporal atoms, dictionary propagation coupled with fewer training iterations and an extended patch-set for dictionary training and image cleaning. It has been dramatically found to improve denoising performance.

The future scope of this study will be the denoising of image sequence can be extended to handle color images using viterbi algorithm which gives better result than denoising each channel separately. This viterbi algorithm can also be used to denoise the impulse noise. The impulse noise can also be called as salt and pepper noise.

\section{REFERENCES}

Aharon, M., M. Elad and A.M. Bruckstein, 2006. On the uniqueness of overcomplete dictionaries and a practical way to retrieve them. J. Lin. Algebra Appl., 416: 48-67.
Boyce, J.M., 1992. Noise reduction of image sequence using adaptive motion compensated frame averaging. ICASSP, 3: 461-464.

Brailean, J.C., R.P. Kleihorst, S. Efstratidis, K.A. Katsaggeleos and R.L. Lagendijk, 1995. Noise reduction filters for dynamic image sequences: A review. Proc. IEEE, 83: 1272-1292.

De Haan, G., 1999. IC for, motion- compensated deinterlacing, noise reduction and picture rate conversion. IEEE Trans. Cosumers Elect., 45: 61 7-624.

Elad, M. and M. Aharon, 2006. Image denoising via learned dictionaries and sparse representation. Proceedings of the International Conference Computer Vision and Pattern Recognition, June 1722, New York, pp: 895-900.

Elad, M. and M. Protter, 2009. Image denoising via sparse and redundant representations. IEEE Trans. Image Process., 18: 27-35.

Jansen, M., 2001. Noise Reduction by Wavelet Thresholding. Springer-Verlag, Berlin.

Kuo, C.J. and C.S. Lin, 1996. Viterbi-based algorithm for side-match vector quantization over noisy channels. IEEE Trans. Commun., 44: 1455-1465.

Pizurica, A., V. Zlokolica and W. Philips, 2003. Noise reduction in video sequences using wavelet-domain and temporal filtering. Proceedings of the SPIE Conference on Wavelet Applications in Industrial Processing, Oct. 27-31, Providence, Rhode Island, USA., pp: 48-59.

Rajpoot, N., Z. Yao and R. Wilson, 2004. Adaptive wavelet restoration of noisy video sequences. Proc. Int. Conf. Image Process., 2: 957-960.

Siu, M. and A. Chan, 2006. A robust viterbi algorithm against impulsive noise with application to speech recognition. Audio Speech Language Process. IEEE Trans., 14: 2122-2133.

Zlokolica, V., A. Pizurica and W. Philips, 2004. Recursive temporal denoising and motion estimation of video. Proc. Int. Conf. Image Process., 3: 1465-1468. 